\title{
Emollients for the prevention of atopic dermatitis
}

\author{
Akash Kothari ${ }^{1}$, Arielle Locke ${ }^{2}$, and Thomas Eiwegger ${ }^{3}$ \\ ${ }^{1}$ University of Toronto \\ ${ }^{2}$ National University of Ireland Galway \\ ${ }^{3}$ The Hospital for Sick Children
}

September 24, 2020

Article Type: News and Views: Groundbreaking Discoveries in Immunology

Title: Emollients for the prevention of atopic dermatitis

Authors: Akash Kothari ${ }^{1}$ (https://orcid.org/0000-0003-1980-161X), Arielle Locke²,

Thomas Eiwegger ${ }^{1,3,4}$ (https://orcid.org/0000-0002-2914-7829)

${ }^{1}$ Translational Medicine Program, Research Institute, Hospital for Sick Children, Toronto, Ontario, Canada

${ }^{2}$ Department of Medicine, National University of Ireland, Galway, Ireland

${ }^{3}$ Division of Immunology and Allergy, Food Allergy and Anaphylaxis Program, The Department of Pediatrics, Hospital for Sick Children, Toronto, Ontario, Canada

${ }^{4}$ Department of Immunology, Faculty of Medicine, University of Toronto, Toronto, Ontario, Canada

Correspondence to: Thomas Eiwegger, MD, Division of Immunology and Allergy, Food Allergy and Anaphylaxis Program, The Department of Paediatrics, Hospital for Sick Children, 555 University Ave, Toronto, Canada, E-mail: thomas.eiwegger@sickkids.ca, Tel.: +1 416-813-7654 ext. 1862

Conflicts of Interest: AK and AL have nothing to disclose. TE reports to act as local PI for company sponsored trials by DBV and sub-investigator for Regeneron, holds grants from Innovation Fund Denmark, CIHR outside the submitted work. He is Co-Investigator or scientific lead in three investigator initiated oral immunotherapy trials supported by the Food Allergy and Anaphylaxis Program SickKids and serves as associate editor for Allergy. He/his lab received unconditional/in-kind contributions from Macro Array Diagnostics and an unrestricted grant from ALK. He holds advisory board roles for ALK.

Financial support: This work was supported by The Hospital for Sick Children, The Food Allergy and Anaphylaxis Program at The Hospital for Sick Children, and The Dr Lorus J And Dr Margery J Milne Scholarship from Victoria University at the University of Toronto.

Statement of Author Contribution: All authors critically reviewed the original articles (references 6 and 7) and wrote the News \& Views: Groundbreaking discoveries in Immunology article. All authors contributed, revised, edited, and approved the final version of the manuscript as submitted and agreed to be accountable for all aspects of the work.

Keywords: emollient, atopic eczema, infancy

Abbreviations : food allergy, FA; filaggrin gene, FLG; atopic dermatitis, AD; transepidermal water loss, TEWL; Barrier Enhancement for Eczema Prevention, BEEP; Preventing Atopic Dermatitis and Allergies, PreventADALL 


\section{Word Count: 997}

Atopic dermatitis/Eczema (AD) is the most common chronic inflammatory skin disease affecting up to $30 \%$ of children. Eczema typically develops during infancy, characterized by pruritus, dry skin, and eczematous dermatitis/skin irritation and often represents the initial manifestation of the so-called atopic march.

Thus, skin barrier dysfunction due to environmental factors and genetic predisposition paired with skin inflammation is linked to AD and consequently food sensitization, food allergy (FA) and progression of the allergic disease complex (Figure 1). ${ }^{1}$ The importance of barrier integrity for AD is supported by filaggrin (FLG). It is integral in maintaining stratum corneum integrity. ${ }^{1}$ Loss of function mutations of FLG gene confer a greater risk of $\mathrm{AD}{ }^{2}$ The association between mutations in the FLG gene and FA independently of $\mathrm{AD}$ is under debate due to conflicting epidemiological data. ${ }^{2}$ However, skin barrier dysfunction may also be relevant at subclinical levels facilitating type-2 skin inflammation which in turn reduces FLG expression. ${ }^{2}$ By connecting the dots between skin barrier dysfunction and food avoidance in the context of environmental food exposure compared to concurrent consumption, the dual-allergen exposure hypothesis has been developed. It is strongly supported by the results of the LEAP study. ${ }^{3}$ The hypothesis suggests that cutaneous allergen exposure and concurrent compromised barrier with skin inflammation while delaying food introduction results in a greater susceptibility for FA. Allergen exposure in such an inflammatory skin context is considered to promote type- 2 inflammation. ${ }^{2}$

Increased transepidermal water loss (TEWL), a surrogate marker of reduced barrier function, precedes $\mathrm{AD}$ development. ${ }^{2} \mathrm{TEWL}$ is lowest at birth and increases within the first two months before plateauing at 6 months, identifying this period as a window of opportunity for preventive interventions. ${ }^{2}$ Pilot studies with paraffin-based emollients suggested a $50 \%$ risk reduction of developing AD (43\% controls vs $22 \%$ emollient treated patients). ${ }^{4,5}$ While applying ointment is a central therapeutic concept for AD treatment, the preventive effect of ointments for $\mathrm{AD}$ and potentially allergic disease development remains to be established via large prospective interventional trials.

Therefore, two prospective, open-label, interventional, randomized preventive trials have attempted to assess the potential of emollients for the primary prevention of AD. These two investigator-initiated studies, the BEEP (Barrier Enhancement for Eczema Prevention) ${ }^{6}$ and the PreventADALL (Preventing Atopic Dermatitis and Allergies) ${ }^{7}$ trial have shown otherwise (Figure 2). Both attempted to create a real-life setting giving parents a lot of control over treatment administration: The BEEP study enrolled 1394 high-risk infants (a first-degree relative with physician diagnosed allergic disease) to receive either a daily paraffin-based emollient or a standardized skin-care regimen within the first 3 weeks of life. ${ }^{6}$ The PreventADALL study studied 2397 population-based infants assessing two interventions: daily petroleum-based emollient usage in the facial area and bathing additives (8-month intervention, minimum four days/week) compared to a food introduction protocol, a combination of both or best skin care practice (controls). ${ }^{7}$ Food introduction consisted of sequential introduction of peanut butter at 3 months of age, with cow's milk, wheat porridge, and scrambled egg sequentially following each week. The primary outcome of BEEP was the development of eczema at two years of life after the one-year intervention that consisted of a daily whole-body application with a good adherence (minimum of application: at least 3-4 days per week in at least two areas: face and neck, arms and legs, or trunk) of $88 \%$ at 3 months, $82 \%$ at 6 months and $74 \%$ at 12 months. ${ }^{6}$ The PreventADALL's primary outcomes were $\mathrm{AD}$ at the age of 12 months and food allergy at age 3 (not reported yet).

In the BEEP study, AD occurred at comparable rates at the age of 2 years $\left(23 \%\right.$ vs $25 \%$, n.s.) ${ }^{6}$ The PreventADALL study also reports null findings for AD prevention via ointment application in a population-based setting (8\% control, $11 \%$ emollient group, $9 \%$ early allergen complementary feeding group, $5 \%$ combined intervention, at year 1). ${ }^{7}$ This study adds an additional component, the value of concurrent early food introduction for the prevention of AD. Of note, the combined intervention of emollient and early food introduction had a tendency to reduce eczema prevalence ( $8 \%$ vs $5 \%) .{ }^{7}$ This finding was not considered clinically meaningful, defined as 1 case of $\mathrm{AD}$ prevented in 14 cases treated $(\Delta 7 \%) \cdot{ }^{7}$ Results raise more questions regarding preventive approaches in the field of atopic diseases than they answer and exemplify the complex nature of 
AD. There are also a number of limitations: the PreventADALL study suffered from limited adherence $(27 \%$ skin intervention ${ }^{7}, 30 \%$ food intervention ${ }^{7}$ ), used an unusual approach when choosing a facial application of the emollient (face only ${ }^{7}$ ), and the very early introduction strategies may have impacted compliance (3 months $\left.{ }^{7}\right)$. Neither of the two studies addressed a key factor which is inflammation. Absence or suppression of inflammation when addressing barrier dysfunction may be key. Another important consideration is that not all emollients are the same. Petroleum-based approaches have been demonstrated to be less effective in reducing TEWL compared to ceramide-based approaches. ${ }^{8}$ Ointment application may have to occur several times per day and at least 5 days per week to affect barrier function. Moreover, poor compliance was not due to adverse/safety effects of the interventions. These limitations await to be addressed in future and ongoing studies (PEBBLES (NCT03409367), CASCADE (NCT03409367), and PACI (UMIN-CTR: UMIN000028043)).

Previous studies identified ointments, especially those of peanut oil origin which comprise largely of lipids and not protein, as a potential facilitator of allergy. ${ }^{9}$ The presence of emollients may facilitate uptake of certain substances, including allergens, in the superficial epithelial cell layers instead of protecting the skin barrier. ${ }^{2}$ It is unclear to what extent this could have played a role in these two studies as different preparations may elicit different effects on the immune system.

Understanding barrier function and inflammation of the skin and the mucosal surfaces early in life is key to design novel preventive interventions and these two studies have added important negative data to design alternative approaches. Thus, usage of petroleum-based emollients specifically and emollient in general as a primary prevention for AD is not supported until further evidence in favor is generated.

\section{References}

1. Eiwegger T, Hung L, San Diego KE, O'Mahony L, Upton J. Recent developments and highlights in food allergy. Allergy . 2019;74(12):2355-2367. doi:10.1111/all.14082

2. Brough HA, Nadeau KC, Sindher SB, et al. Epicutaneous sensitization in the development of food allergy: What is the evidence and how can this be prevented? Allergy . May 2020:all.14304. doi:10.1111/all.14304

3. Du Toit G, Roberts G, Sayre PH, et al. Randomized Trial of Peanut Consumption in Infants at Risk for Peanut Allergy. N Engl J Med . 2015;372(9):803-813. doi:10.1056/NEJMoa1414850

4. Horimukai K, Morita K, Narita M, et al. Application of moisturizer to neonates prevents development of atopic dermatitis. J Allergy Clin Immunol . 2014;134(4):824-830.e6. doi:10.1016/j.jaci.2014.07.060

5. Simpson EL, Chalmers JR, Hanifin JM, et al. Emollient enhancement of the skin barrier from birth offers effective atopic dermatitis prevention. J Allergy Clin Immunol . 2014;134(4):818-823. doi:10.1016/j.jaci.2014.08.005

6. Chalmers JR, Haines RH, Bradshaw LE, et al. Daily emollient during infancy for prevention of eczema: the BEEP randomised controlled trial.Lancet . 2020;395(10228):962-972. doi:10.1016/S0140-6736(19)329848

7. Skjerven HO, Rehbinder EM, Vettukattil R, et al. Skin emollient and early complementary feeding to prevent infant atopic dermatitis (PreventADALL): a factorial, multicentre, cluster-randomised trial.Lancet . 2020;395(10228):951-961. doi:10.1016/S0140-6736(19)32983-6

8. Sindher S, Alkotob SS, Shojinaga MN, et al. Pilot study measuring transepidermal water loss (TEWL) in children suggests trilipid cream is more effective than a paraffin-based emollient. Allergy . March 2020:all.14275. doi:10.1111/all.14275

9. Lack G, Fox D, Northstone K, Golding J. Factors Associated with the Development of Peanut Allergy in Childhood. N Engl J Med . 2003;348(11):977-985. doi:10.1056/NEJMoa013536

Figure 1. Environmental and genetic factors affecting skin barrier dysfunction and inflammation, leading to the development of the allergic disease complex. Epicutaneous allergen exposure happens due to the presence 
of food allergens in the environment. In the absence of oral ingestion this cutaneous exposure in patients with eczema is thought to promote allergic sensitization, resulting in a greater susceptibility to develop food allergy. It is unknown whether emollient usage may promote barrier integrity and affect the interplay between barrier impairment, skin inflammation, and ultimately prevent atopic dermatitis development.

Figure 2. Two prospective interventional trials assessed the preventive capacity of emollient usage on the development of atopic dermatitis at 24 months (BEEP) and at 12 months (PreventADALL). The BEEP study recommended a daily paraffin-based whole-body emollient vs. a standardized skin-care regimen within the first 12 months of life in 1394 high-risk infants and assessed the rate of AD at 24 months. The PreventADALL study studied 2397 population-based infants assessing the impact of petroleum-based emollient usage in the facial area in combination with bathing additives compared to an early food introduction protocol, a combination of both interventions, or best skin care practice. No superiority of any of these interventions over best skin practice regarding the prevention of $\mathrm{AD}$ was observed. AD: atopic dermatitis

\section{Hosted file}

Figure 1 Kothari et al.pptx available at https://authorea.com/users/361343/articles/482756emollients-for-the-prevention-of-atopic-dermatitis

\section{Hosted file}

Figure 2 Kothari et al.pptx available at https://authorea.com/users/361343/articles/482756emollients-for-the-prevention-of-atopic-dermatitis 